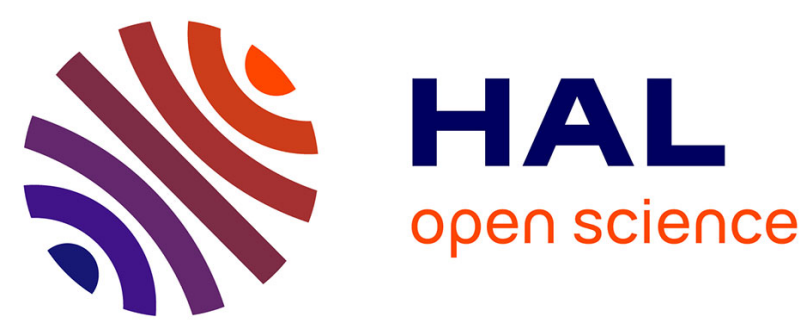

\title{
A Stochastic Framework for Neuronal Morphological Comparison: Application to the Study of imp Knockdown Effects in Drosophila Gamma Neurons
}

Agustina Razetti, Xavier Descombes, Caroline Medioni, Florence Besse

\section{- To cite this version:}

Agustina Razetti, Xavier Descombes, Caroline Medioni, Florence Besse. A Stochastic Framework for Neuronal Morphological Comparison: Application to the Study of imp Knockdown Effects in Drosophila Gamma Neurons. Ana Fred; Hugo Gamboa. Biomedical Engineering Systems and Technologies, 690, Springer, pp.145-166, 2017, CCIS, 10.1007/978-3-319-54717-6_9 . hal-01502735

\section{HAL Id: hal-01502735 \\ https://hal.inria.fr/hal-01502735}

Submitted on 7 Apr 2017

HAL is a multi-disciplinary open access archive for the deposit and dissemination of scientific research documents, whether they are published or not. The documents may come from teaching and research institutions in France or abroad, or from public or private research centers.
L'archive ouverte pluridisciplinaire HAL, est destinée au dépôt et à la diffusion de documents scientifiques de niveau recherche, publiés ou non, émanant des établissements d'enseignement et de recherche français ou étrangers, des laboratoires publics ou privés. 


\title{
A stochastic framework for neuronal morphological comparison: application to the study of imp knockdown effects in Drosophila Gamma neurons
}

\author{
Razetti A. ${ }^{1}$, Descombes X. ${ }^{2}$, Medioni C. ${ }^{3}$, and Besse F. ${ }^{3}$ \\ ${ }^{1}$ University of Nice Sophia Antipolis, I3S, 2000 Route des Lucioles, Sophia Antipolis, France \\ arazetti@unice.fr \\ ${ }^{2}$ Inria, CRISAM, 2003 Route des Lucioles, Sophia Antipolis, France \\ xavier.descombesdinria.fr \\ ${ }^{3}$ Institute of Biology Valrose, University of Nice Sophia Antipolis, Parc Valrose, Nice, France \\ \{caroline.medioni, florence.besse\} dunice.fr
}

\begin{abstract}
In order to reach their final adult morphology, Gamma neurons in Drosophila brain undergo a process of pruning followed by regrowth of their main axons and branches called remodelling. The mRNA binding protein Imp was identified to play a fundamental role in this process. One of Imp targets, profilin mRNA, encodes for an actin regulator that has been shown to be involved in axon remodelling. In this paper we intend to further understand the role of Imp and the importance of profilin mRNA expression regulation during remodelling. To do so, we propose a stochastic framework to exhaustively compare the adult morphology between wild type (WT), imp knockdown (Imp) and imp knockdown rescued by Profilin (Prof Rescue) neurons. Our framework consists in i) the selection of the main neuron morphological features, ii) their stochastic modelling and parameter estimation from data and iii) a maximum likelihood analysis for each individual neuron to quantitatively assess the similarity or difference between groups. Thanks to this framework we show that imp mutant neurons can be divided in two phenotypical groups with a different aberrancy degree, and that profilin overexpression partially rescues the main axon and branch development thereby it reduces the proportion of neurons with the strongest remodelling phenotype.
\end{abstract}

Keywords: Gamma neurons, Remodelling, Stochastic models, Maximum likelihood analysis.

\section{Introduction}

Gamma neurons in Drosophila brain mushroom body are in charge of high functions such as olfactory learning and memory [1]. Mutations affecting their adult shape cause several behavioral dysfunctions [2].

During metamorphosis, gamma neurons go through a process of pruning -where the main part of their axons and dendrites is lost-followed by regrowth of the main axon

adfa, p. 1, 2011.

(C) Springer-Verlag Berlin Heidelberg 2011 
and branches, resulting in the establishment of the adult shape [3]. The correct development of this process gives rise to well-formed and functional adult neurons.

This study is focused on the role of the mRNA binding protein Imp in the remodelling process. Medioni et al. [4] have shown that even though Imp is not essential during the initial axonal growth of gamma neurons, it is necessary during their remodelling. They report that, in adults, $~ 50 \%$ of imp mutants display shorter axons than wild types (WT) and fail to reach their target. imp mutants also exhibit an overall loss of branch number and complexity [4].

Molecular and genetic analysis have further shown that profilin mRNA, which encodes an actin cytoskeleton regulator [5,6,7], is a direct and functional target of Imp and both are key regulators of the Drosophila gamma neuron axonal remodelling process, acting on the same molecular pathway. Interestingly, the overexpression of profilin in imp mutants seems to partially rescue the main axon length, but not the branch complexity. These results suggest that Imp controls axonal extension during remodelling at least partly by regulating profilin mRNA expression. However they also suggest that the branching process may be dependent on the regulation of other imp mRNA targets, yet to be identified.

In this paper, we intend to further understand the role of Imp and the importance of profilin mRNA regulation during remodelling, based on a deep analysis of the impact of imp knockdown, and its rescue by profilin overexpression, in neuron adult morphology (Figure 1). To do so, an exhaustive morphological comparison of the main features between WT and the mutated axons is needed. We propose in this work a stochastic framework to accurately achieve this comparison, that can be summarized in three steps: i) selection of relevant morphological features describing the data, ii) stochastically model the behavior followed by each of the chosen morphological features and estimate the associated parameters to each model from the data (for WT and mutant groups) and iii) perform a maximum likelihood analysis for each individual neuron (classification) -considering the features separately and altogether- to quantitatively assess the global similarity or difference between groups (through the classification performance). We also developed statistical tests under null hypothesis between the neuron groups for each morphological feature to enrich the analysis. This approach provides both a biological interpretation and a quantification of resemblance between biological samples, detecting differences as well as similarities between the groups. This framework is general and can be applied to model and characterize any kind of neurons (Figure 2).

Because effects of imp knockdown and rescue with Profilin can be identified in the main axon as well as in the branch development or separately, we considered both structures separately. The four chosen main morphological features are: "main axon length", "main axon shape", "first order branch distribution along the main axon" and "branch length distribution". To measure these features, we segmented a set of images corresponding to each neuron group (wild type or mutated) to obtain a numeric 3D treeshaped skeleton representing the morphology of each neuron. We then measured the feature values using homemade software. The image segmentation as well as the measurement of each feature are described in the following sections (Figure 2).

Neuron morphological automatic classification has already been addressed in the bibliography. Kong et al. [8] proposed an unsupervised clustering of ganglion cells in 
the mouse retina by the k-means algorithm in order to define cell types. They initially disposed of 26 morphological parameters and found out that clustering with only three of them was the most effective way. Guerra et al. [9] established the advantage of applying supervised classification methods regarding morphological feature based classification to distinguish between interneurons and pyramidal cells. They also conclude that reducing the number of features to an optimal number outperforms the classical approach of using all the available information. Lopez-Cruz et al. [10] built a consensus Bayesian multinet representing the opinions of a set of experts regarding the classification of a pool of neurons. The morphological parameters chosen by each expert to make their decisions are not considered. A different approach was proposed by Mottini et al. [11] which consists on classifying different neuron types by reducing them to trees and calculating a distance, combining geometrical and topological information.

The different published approaches intend to accurately discriminate between different types of neurons, considering misclassification as a methodological error and consequently developing techniques to avoid these cases. However, similarities between populations are not necessarily to be excluded as they may reflect the properties of biological samples and provide useful information for their characterization. Furthermore, these methods do not intend to understand which morphological characteristic is discriminant between different species and at which level. A deeper multi-criteria statistical analysis is thus required. Our approach allows to assess the similarities and dissimilarities between the populations for each chosen morphological feature separately as well as considering them all together for a global analysis. Neurons are treated individually through the resemblance analysis as well as globally within the studied groups through the statistical comparisons, achieving an exhaustive analysis.

In the next sections, we introduce each one of the features followed by its stochastic model. Next we present the results of the classification combining different criteria, which allows to finally deduce the morphological changes induced by the studied mutations.

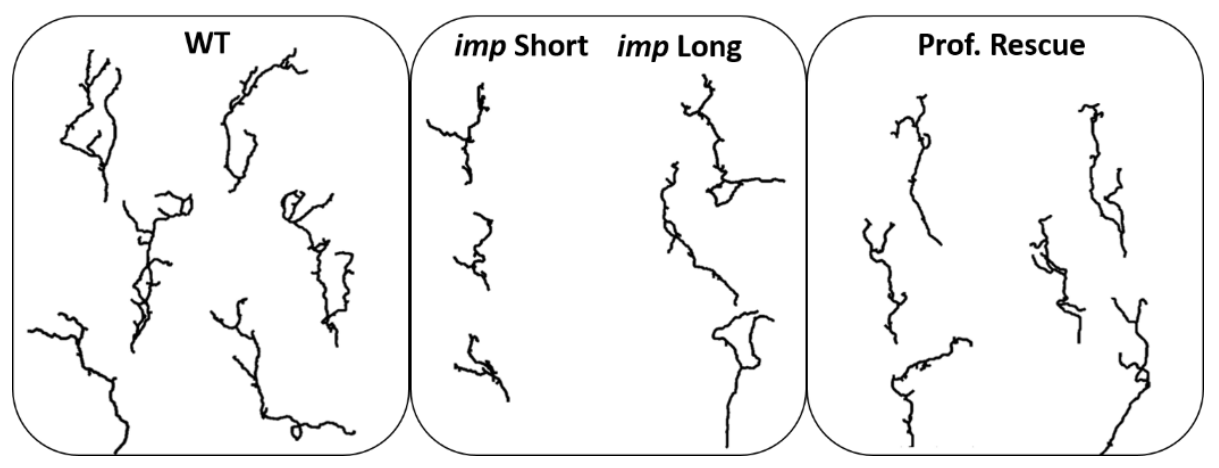

Fig. 1. Representation of the morphology of each one of the groups (in order: Wild type, imp mutant and imp mutant rescued by Profilin). imp mutants are divided into short and long species (named Imp Sh and Imp L respectively) as both phenotypes are equally observed [4]. 
Stochastic framework

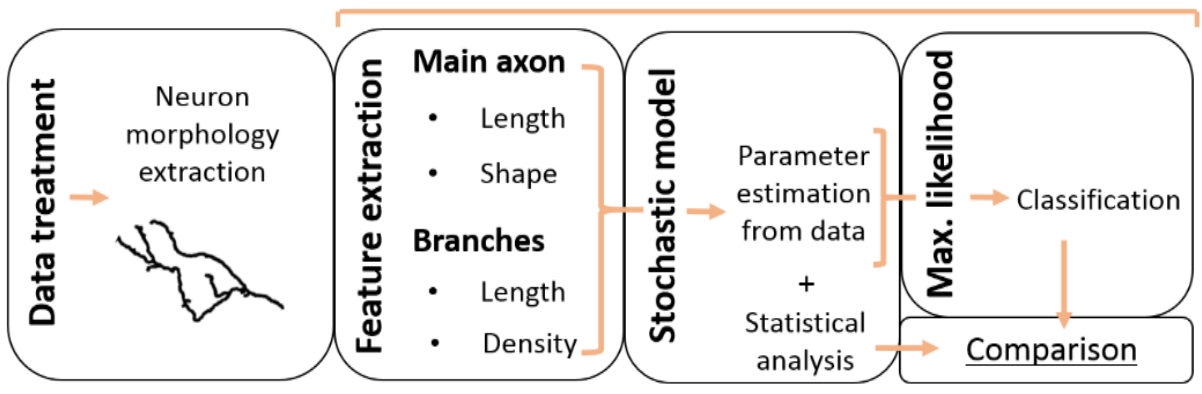

Fig. 2. Scheme of the proposed stochastic framework for the comparison of neuron morphologies between groups.

\section{Data}

\subsection{Images}

We used 3D images taken with a confocal microscope. Each set of images shows the distal part of an axonal tree at adult stage (Figure 3). Single axons are labelled by GFP using the MARCM technique [12], which allows to image a single mutated (or wild type) neuron in a wild type environment. The database we used for this study consists in 46 wild type images, $48 \mathrm{imp}$ mutants and $15 \mathrm{imp}$ mutants rescued by profilin overexpression.

The voxel size varies among the images and is anisotropic in the $\mathrm{Z}$ axis. The voxel length in $\mathrm{Z}$ is between 5 and 12 times its length in $\mathrm{X}$ and $\mathrm{Y}$, which varies from 0.09 to $0.15 \mu \mathrm{m}$ (Figure 3).
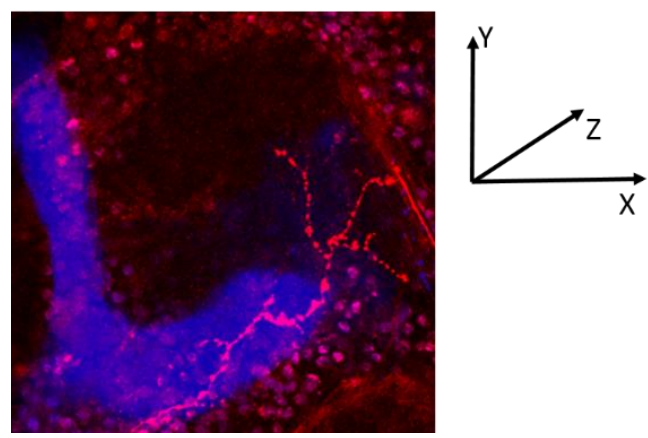

Fig. 3. Maximum intensity $\mathrm{Z}$ projection showing a wild type axon and the morphology of the mushroom body. 


\subsection{Segmentation}

To avoid artificial jumps along the $\mathrm{Z}$ axis due to image anisotropy, we applied a simple quadratic interpolation algorithm included in FIJI (the open source image analysis software developed by NIH, Maryland, USA) [13].

An automatic segmentation of these images is still not available regarding our needs due to a noisy background and poorly defined and non-continuous neuron trace. Therefore we segmented the images with the open software Neuromantic [14], specially developed to segment 2 or $3 \mathrm{D}$ neurons manually or semi-automatically. As output we obtained a set of points along the main axon and branches that we connected using a Bresenham-inspired 6-connectivity algorithm. We chose this connectivity to keep further measurements simple. After this process we obtained a tree-like set of numeric 3D curves that describe the morphology of each neuron (Figure 4).

To ensure all the neurons to be similarly oriented we rotated the images and aligned them with respect to reference structures (i.e. medial and dorsal lobes of the mushroom body, Figure 3). We only considered a rigid transformation to avoid axon deformation. Conserved morphology was preferred rather than more accurate spatial location.

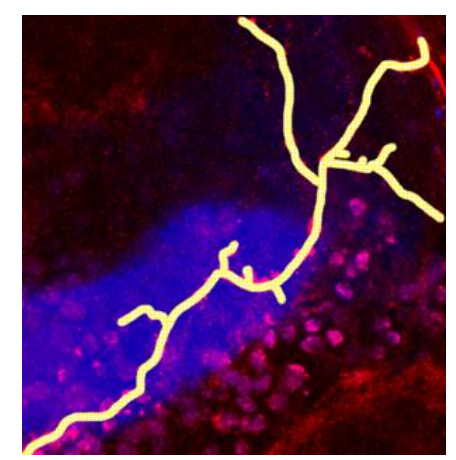

Fig. 4. Zoom of the Z projected image showed in Figure 3, where the neuron has been segmented to obtain a tree-like set of numeric $3 \mathrm{D}$ curves.

\subsection{Tree hierarchy}

When studying their morphology it is necessary to understand how neurons are structured i.e. main axon and first, second, third (etc.) order branches (the neuron cell body and dendrites are not considered in this study). To accurately label each path of the tree that represents each neuron, we developed an automatic recursive algorithm capable of processing trees of any order. It starts by taking the whole tree and selects the main axon. After this process a number of independent subtrees is formed. During the following steps each of the subtrees is analyzed to assign their main path and so on, until no more untagged segments are left. In each step, the main path of the subtree is assigned following the same criteria used by experts when done visually: total length, directionality and sense coherence. To achieve this, we consider the points for each path between the root and the leaves of the tree (i.e. the whole neuron) or subtree and 
calculate a linear regression obtaining a straight guideline, which will determine directionality and sense coherence. For each path in the analyzed tree/subtree, a cost function is computed that depends on the distance between each point in the path and the guideline (directionality), the parallelism between them (accounting for the sense coherence of the path) and the path total length. Finally the path that minimizes this cost function is selected as "main axon" in the case of the whole tree (first step), or "main branch" in the case of the different subtrees (Figure 5).

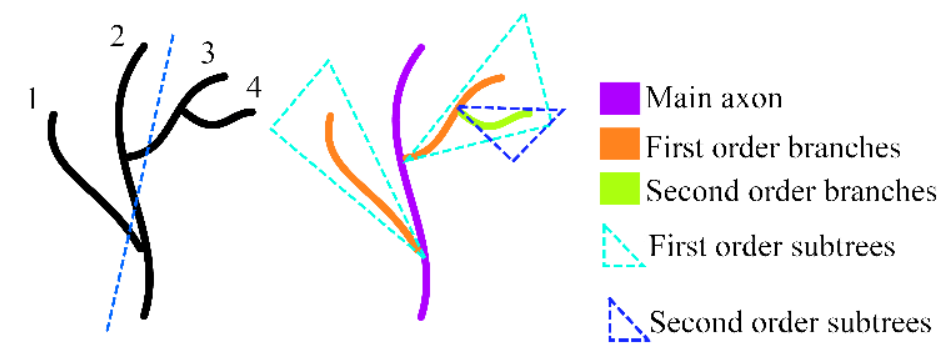

Fig. 5. Scheme of the three-hierarchy algorithm. For a given tree, the guideline is calculated as the linear regression of all the points in the tree, followed by the cost function for each possible path (1-4). The one that minimizes is assigned as "main axon" (here path 2). The algorithm is applied recursively to each subtree resulting in the hierarchy of the entire tree.

\section{Model development}

After the processes described in the previous section, our data is composed of 3D skeletons of neurons where each unitary 6-connected segment is described by its round coordinates or pixels. Taking this simple geometrical description of the neuron into account, we defined the main morphological features that may best describe and discriminate the individuals: the main axon length and sinuosity, as well as the branch density and length distribution. In the following sections we develop the probabilistic models chosen to describe each feature, estimate their parameters and compute associated statistical tests under null hypothesis between the different groups (WT: wild type neurons that are used as controls, Imp: imp knockdown neurons, reported to be morphologically aberrant in the literature, and Prof Rescue: imp mutants with an overexpression of profilin, known to partially suppress the imp mutant phenotype) (Figure 1). Besides, we derive the likelihood of each model that is used in Section 4.

\subsection{Main axon length}

The main axon length was measured taking the total amount of pixels in the corresponding path and multiplying it by the pixel size $(\mu \mathrm{m})$. The length distribution was modelled as Gaussian, and the mean and standard deviation for each group $X\left(\mu_{m . a}^{X}, \sigma_{m . a}^{X}\right)$ were calculated from data. We observed the bimodal behaviour in the Imp group reported by Medioni et al. [4] (Figure 6). Therefore, in order to make a more accurate modelling 
of this parameter, we separated imp mutant neurons into two groups -neurons with long axons (Imp L) and neurons with short axons (Imp Sh)- using the k-means algorithm. $54 \%$ of the neurons were assigned to Imp Sh and $46 \%$ to Imp L, consistent with the percentage reported by Medioni et al. [4]. Figure 6 shows the main axon length histograms for each group, Imp divided into Imp L and Imp Sh. We decided to keep this division of the Imp group (Imp L and Imp Sh) through all the analysis in this paper, in the attempt to detect other morphological differences between the two subgroups.

To assess which groups present significant differences regarding the main axon length, non-parametric Kruskal Wallis tests were carried out between all the possible pairs of groups (Table 1). We chose this test for the sake of consistency, as it can be applied to analyze all the features independently of each model. For $p$ values inferior to $5 \%$, we consider the null hypothesis that both distributions are the same can be rejected. Thus, the only pair not presenting a significant difference is WT vs. Imp L. It is interesting to highlight also that Prof Rescue distribution lies in between the distributions for Imp L and Sh and is more similar to Imp L, meaning it does not present extremely short axons.

The likelihood of a given neuron $n$ of length $l_{n}$ to belong to a given group $X$ is defined by the Normal probability density function

$$
L_{l}\left(l_{n} \mid n \in X\right)=P\left(l_{n} \mid n \in X\right)=\frac{1}{\sigma_{m \cdot a}^{X} \sqrt{2 \pi}} e^{\frac{-\left(l_{n}-\mu_{m \cdot a}^{X}\right)^{2}}{2 \sigma_{m \cdot a}^{X}}} .
$$

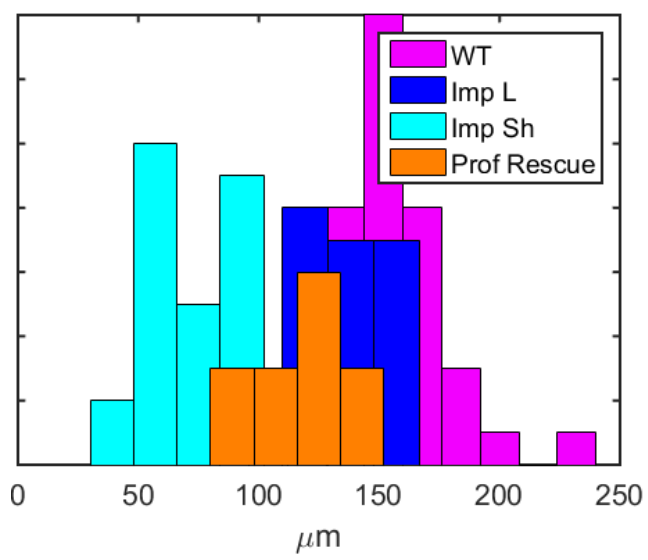

Fig. 6. Main axon length distributions for each biological sample.

Table 1. $p$ values from the non-parametric Kruskal Wallis test comparing the main axon length between the studied groups.
$\operatorname{Imp~L}$
Imp Sh
Prof Rescue

WT

0.1219

5.0098E-12

0.000144

Imp L

3.2627E-09

0.0013 


\subsection{Main axon morphology}

To define the shape model, we considered as random variable the unit vector $\vec{x}_{t}$ that accounts for the shift of the axon tip between $t-1$ and $t$. Because we used the 6-connectivity and backwards moves are not allowed, each $\vec{x}_{t+1}$ (shift of the axon tip between $t$ and $t+1$ ) can take five different values, as shown in Figure 7. Assuming the main axon development follows a second order Markov property, we have $[15,16]$

$$
P\left(\vec{x}_{t+1} \mid \vec{x}_{i} i \leq t\right)=P\left(\vec{x}_{t+1} \mid \vec{x}_{t}, \vec{x}_{t-1}\right) .
$$

The shape model is then completely defined by the conditional probabilities $P\left(\vec{x}_{t+1} \mid \vec{x}_{t}, \vec{x}_{t-1}\right)$. There are 30 possible combinations of the two unit vectors $\left[\vec{x}_{t}\right.$, $\left.\vec{x}_{t-1}\right]$ and each of these combinations has five possible future jumps $\vec{x}_{t+1}$, giving a total of 150 possible transitions in $t+1$, each of them with probability $P_{i}$ (conditionally to $\left.\left[\vec{x}_{t}, \vec{x}_{t-1}\right]\right)$. The order of the Markov chain was chosen in order to combine a discriminative efficiency between similarly shaped axons and a reasonable combinatorial to robustly estimate the conditional probabilities.

Figure 7 presents two basic configurations of a pair of unit vectors $\left[\vec{x}_{t}, \vec{x}_{t-1}\right]$ and their corresponding five possible $\vec{x}_{t+1}$. The one on the left depicts one of the six possible cases where the vectors $\vec{x}_{t}$ and $\vec{x}_{t-1}$ are aligned. The second configuration exemplifies the 24 cases where the vectors $\vec{x}_{t}$ and $\vec{x}_{t-1}$ are not aligned.

We estimate the conditional probabilities from data using the empirical estimator (3), where $\#_{n}$ accounts for the number of times the $\mathrm{n}^{\text {th }}$ configuration of three unit vectors $\left[\vec{x}_{t+1}, \vec{x}_{t}, \vec{x}_{t-1}\right]$ appears.

$$
P_{5 s+j}=\frac{\#_{5 s+j}}{\sum_{k=1}^{5} \#_{5 s+k}}, \begin{gathered}
j=1, \ldots, 5 \\
s=0, \ldots, 29
\end{gathered}
$$

We performed the Kruskal Wallis non-parametric test between populations for each $P_{i}, 1 \leq i \leq 150$. Table 2 shows the number of parameters $P_{i}$ that present a $p$ value inferior to $5 \%$ between each pair of populations. In addition, regarding the possible two past unit vectors $\left[\vec{x}_{t}, \vec{x}_{t-1}\right]$, the results of the estimation show that all the groups share the six most frequent configurations, representing together between 65 and $76 \%$ of the total.

The computation of the Markov chain likelihood appears to lack of robustness to compare populations. This can be explained by the limited length of the axons in pixels $(\sim 1500)$ and the combinatorial of the problem (150 conditional probabilities). Indeed, some of the three vector configurations, even though with non-zero probability, may not appear in the learning sample. When this is the case, if the axon to classify does present at least one time this configuration the likelihood becomes zero. This means that the likelihood is extremely sensible to fluctuations in the presence of low probable events, which is statistically inevitable with the size of our data. To overcome this inconvenience and add robustness to the likelihood analysis, an original approach was 
applied (4). We assume the 30 probability distributions $P\left(\vec{x}_{t+1} \mid \vec{x}_{t}, \vec{x}_{t-1}\right)$ as independent, and defined a multinomial Bernoulli distribution [17] for the variable $\vec{x}_{t+1}$ for each given $\left[\vec{x}_{t}, \vec{x}_{t-1}\right]$.

For each neuron $\mathrm{n}$, the likelihood of each group $\mathrm{X}$ according to the shape model of $\mathrm{X}, P_{i, X}$, and the frequencies of appearance of three unit vectors corresponding to $\mathrm{n}$, $\#_{i}$, is then defined as follows

$$
\begin{gathered}
L_{s h}\left(\#_{1}-\#_{150} \mid n \in X\right)=P\left(\#_{1}-\#_{150} \mid n \in X\right) \\
=\prod_{s=1}^{30}\left(\left(\begin{array}{c}
N_{s} \\
\#_{k+1}
\end{array}\right) P_{k+1}{ }_{k+1}^{\#_{k+1}}\left(\begin{array}{c}
N_{s}-\#_{k+1} \\
\#_{k+2}
\end{array}\right) P_{k+2}{ }^{\#_{k+2}}\left(\begin{array}{c}
N_{s}-\#_{k+1}-\#_{k+2} \\
\#_{k+3}
\end{array}\right)\right. \\
\left.P_{k+3}{ }^{\#_{k+3}}\left(\begin{array}{c}
N_{s}-\#_{k+1}-\#_{k+2}-\#_{k+3} \\
\#_{k+4}
\end{array}\right) P_{k+4}{ }^{\#_{k+4}} P_{k+5}{ }^{\#_{k+5}}\right), \\
k=5(s-1), \quad N_{s}=\sum_{j=1}^{5} \#_{5(s-1)+j} .
\end{gathered}
$$

Table 2.Number of parameters with $\mathrm{p}<0.05$ for the non-parametric Kruskal Wallis test.

$$
\text { Imp L Imp Sh Prof Rescue }
$$

WT

Imp L

Imp Sh

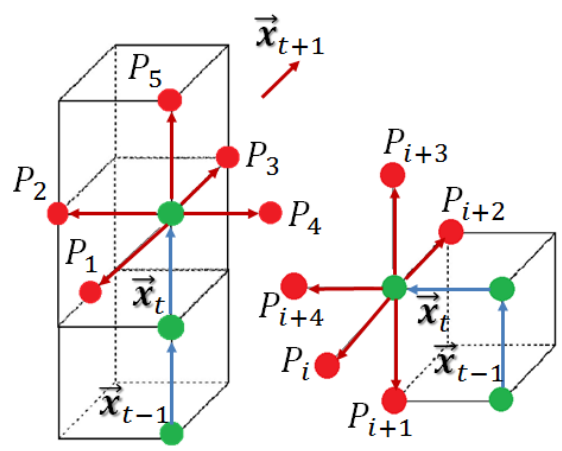

Fig. 7. Two examples of three vector configurations on a 3D 6-connected path. Each future direction has a probability of occurrence conditioned by the present and past directions and is numbered from 1 to 150 (30 possible configurations for past plus present and 5 possible future configurations for each of them). 


\subsection{Branch density}

We propose a model that describes the branching point distribution independently of the axon length and that is based on the biological process of interstitial branch formation during development. This process can be described in three simple steps (Figure 8): A. the main axon grows following particular external and internal guiding cues. B. When the growth cone senses external guiding cues indicating the formation of an interstitial branch, the main axon decreases its growing speed until it totally stops while it accumulates molecular material in its tip. C. After some time the main axon continues growing following its particular cues, leaving the accumulated material in a specific zone of its shaft. The left material has been organized into an independent growing tip and starts elongating an interstitial branch towards its particular target [18].

Modelling this process becomes initially unreachable as none of two main actors (growing rate, guiding cues presence) can be measured from the adult stage static images available as data. Regarding these limitations, we propose a model to mimic this dynamic process from our static data. We focused our study on the behavior of the axon growing rate $v$, starting with a certain initial speed $v_{o}$ and evolving until $v=0$, when a new branch point appears.

We can measure the number $k$ of pixels between every two successive branching points along the main axon of a segmented neuron. Then, we suppose that each of this pixels represents a differential progress in the axonal growth where, during development, the axon had a certain growing rate $v$. Our model assumes random decreases in speed which we call $\Delta v$, with a probability of occurrence $p$. When a certain number of decreases $\Delta v$ occurs, the speed $v$ equals zero thus the growing tip stops, allowing the material needed to form a branch to accumulate. After some time the process starts again, with initial speed $v_{0}$.

Because at each one of the $k$ pixels a decrease in $v$ may or not happen, we describe the problem using a Bernoulli probability distribution [19] where each success means the occurrence of a decrease in speed $\Delta v$. We consider that the growing rate goes to zero after $A+1$ steps of speed decreasing. The probability to reach $v=0$ after $k$ steps is given by an accumulation process and is written as follows:

$$
P(k)=\left(\begin{array}{c}
k-1 \\
A
\end{array}\right) p^{A+1}(1-p)^{k-A-1} .
$$

Equation (5) gives the probability of having $A$ successes in $k-1$ trials and a success in the $k^{\text {th }}$ trial. This means that the axon tip decreases its speed $A$ times before stopping completely (which happens in $A+1$ ), or equivalently that the length between two branching points is $k$ (Figure 9). Thus, our accumulation Bernoulli-based, time-mimicking branching point distribution model has two parameters, $A$ and $p$, to be estimated from data. Knowing all the distances $k_{i}$ between successive branching points for every axon in each group, we can calculate their mean and variance $\mu_{k}$ and $\sigma^{2}{ }_{k}$. From

$$
\mu_{\mathrm{k}}=\sum_{i} k_{i}\left(\begin{array}{c}
k_{i}-1 \\
A
\end{array}\right) p^{A+1}(1-p)^{k_{i}-A-1}
$$

and

$$
\sigma_{\mathrm{k}}^{2}=\sum_{i} k_{i}{ }^{2}\left(\begin{array}{c}
k_{i}-1 \\
A
\end{array}\right) p^{A+1}(1-p)^{k_{i}-A-1}-\mu_{k}^{2}
$$


it can be shown that $\mu_{\mathrm{k}}(A, p)$ and $\sigma_{\mathrm{k}}^{2}(A, p)$ have the simple forms

$$
\mu_{\mathrm{k}}(A, p)=\frac{A}{p} ; \sigma_{\mathrm{k}}^{2}(A, p)=\frac{(1-p) A}{p^{2}}
$$

allowing to easily estimate $A$ and $p$ from data (proof described in Appendix). Once $A$ and $p$ are estimated, $A$ needs to be rounded as it has to be an integer. Then $p$ can be recalculated knowing the value of $A$ as

$$
p=\frac{\sqrt{A\left(\mu_{\mathrm{k}}+\sigma^{2}{ }_{\mathrm{k}}\right)}}{\mu_{\mathrm{k}}+\sigma_{\mathrm{k}}^{2}} .
$$

The number $A+1$ of needed $\Delta v$ in order to form a branch, and $p$ their probability to happen will define each axonal group regarding their branch density.

Tables 3 and 4 present the resulting values of the parameters for each group and the $p$ values from the non-parametric Kruskal Wallis test comparing all the distances between two consecutive branches $k_{i}$ among neuron groups, respectively.

While each group has the same value of $A$, Imp Sh presents the highest value of $p$. This means that $\Delta v$ occurrence is more probable, thus it takes less time to reach $v=0$, and consequently it is the most branched group. This difference is significant $(p<$ 0.05 ) between Imp Sh and every other group.

To calculate the likelihood of each neuron $n$ to belong to the group $X$ regarding this model, we use the Binomial probability density function considering the distances between each pair of branches $k_{n, m}$ independent between them, obtaining

$$
\begin{aligned}
& L_{b p}\left(k_{n, m} \mid n \in \mathrm{X}\right)=P\left(k_{n}=\left\{k_{n, 1}, \ldots, k_{n, M}\right\} \mid n \in \mathrm{X}\right)= \\
& \prod_{\mathrm{m}=1}^{\mathrm{M}} P\left(k_{n, m} \mid n \in \mathrm{X}\right)=\prod_{\mathrm{m}=1}^{\mathrm{M}}\left(\begin{array}{c}
k_{n, m}-1 \\
A_{X}
\end{array}\right) p_{X}{ }^{A_{X}+1} p^{k_{n, m}-1-A_{X}}
\end{aligned}
$$

where $\mathrm{M}$ is the total number of pairs of branches.

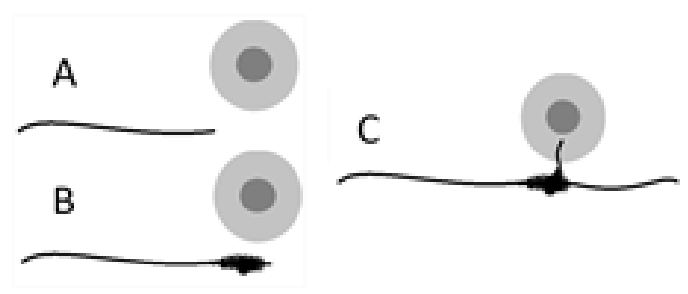

Fig. 8. Interstitial branch formation during axonal development described schematically in three main steps, adapted from Szebenyi et al. [18]. A. the main axon grows. B. When the growth cone senses external guiding cues indicating to branch, the growth speed is decreased until it stops. C. After some time the main axon continues growing, leaving accumulated material in a specific zone of its shaft. The left material has been organized into an independent growing tip and starts elongating an interstitial branch towards its particular target. 


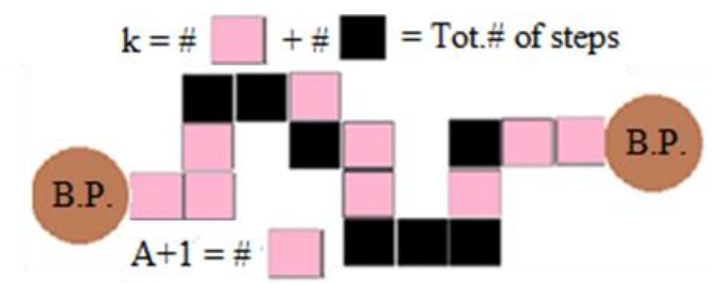

Fig. 9. 2D 4-connected path showing an axonal trajectory between two branching points (3D not shown for simplicity). Light colored pixels depict $\Delta v$ and occur with a probability $p$, decreasing the growing rate. When the number of light colored pixels equals $A+1, v=0$ and a new branching point appears.

Table 3. $p$ values from the non-parametric Kruskal Wallis test comparing the distances in pixels between consecutive branches between the studied groups.

$\begin{array}{cccc} & \text { Imp L } & \text { Imp Sh } & \text { Prof Rescue } \\ \text { WT } & 0.9398 & 4.20 \mathrm{E}-03 & 0.5704 \\ \text { Imp L } & & 2.16 \mathrm{E}-02 & 0.6478 \\ \text { Imp Sh } & & & 1.32 \mathrm{E}-02\end{array}$

Table 4. Value of the parameters $A$ and $p$ describing the branching point distribution.

$\begin{array}{cccc} & A & p & p \text { for } A=1 \\ \text { WT } & 1.2 & 0.0087 & 0.0078 \\ \text { Imp L } & 1.0 & 0.0068 & 0.0067 \\ \text { Imp Sh } & 0.9 & 0.008 & 0.0084 \\ \text { Prof Rescue } & 1.2 & 0.0074 & 0.0068\end{array}$

\subsection{Branch length distribution}

To study the branch length distribution within the neuron groups, we established four length categories $(\mu m) ; L_{1}:(0,1], L_{2}:(1,5], L_{3}:(5,10]$ and $L_{4}:(10, \infty)$ following Tessier and Broadie study [20]. The length was measured in the same way as described for the main axon, and branches of all levels were taken into account. The probability distribution modelling the relative amount of branches within these length categories and for each group was considered as Gaussian. For each group of axons $X$ we calculated the mean and standard deviation $\left(\mu_{b i}^{X}, \sigma_{b i}^{X}\right), 1 \leq i \leq 4$ of the relative number of branches corresponding to each length category per axon $b_{1}-b_{4}$ (i.e. number of branches in each length category normalized by the total number of branches, per axon).

To know between which groups and for which length category the differences displayed in Table 5 are significant, we performed the Kruskal Wallis non-parametric test for the four length groups. Significant results $(p<0.05)$ are only present in $L_{2}$ and $L_{4}$ categories. The $p$ values are shown in Table 6 and Table 7 . 
Imp L presents significantly more branches in $L_{2}$ than any other group while WT has a bigger proportion of $L_{4}$ branches than Imp L and Sh, but not Prof Rescue. For further analysis, we took only the discriminant categories $L_{2}$ and $L_{4}$.

To calculate the likelihood of each neuron $n$ with each group $X$ regarding the branch length distribution in $L_{2}$ and $L_{4}-b_{n, 2}$ and $b_{n, 4^{-}}$we considered a bivariate Gaussian distribution with mean $\vec{\mu}_{b}^{X}=\left(\mu_{b 2}^{X}, \mu_{b 4}^{X}\right)$ and $\Sigma^{X}$ the covariance matrix between $b_{2}^{n}$ and $b_{4}^{n}$.

$$
L_{b l}\left(\vec{b}_{n} \mid n \in \mathrm{X}\right)=P\left(\vec{b}_{n} \mid n \in \mathrm{X}\right)=\frac{1}{2 \pi \sqrt{\left|\Sigma^{X}\right|}} e^{-\frac{1}{2}\left(\vec{b}_{n}-\vec{\mu}_{b}^{X}\right)^{T} \Sigma^{X^{-1}}\left(\vec{b}_{n}-\vec{\mu}_{b}^{X}\right)},
$$

where $\left|\Sigma^{X}\right|$ is the determinant of the covariance matrix $\Sigma^{X}$.

Table 5. Branch length distribution by length and neuron group (\%).

$\begin{array}{ccccc} & L_{1} & L_{2} & L_{3} & L_{4} \\ \text { WT } & 10.6 & 49.2 & 11.7 & 28.5 \\ \text { Imp L } & 8.4 & 66.5 & 10.1 & 15 \\ \text { Imp Sh } & 19.8 & 48.2 & 14.5 & 17.5 \\ \text { Prof Rescue } & 19.5 & 48.3 & 10.2 & 22\end{array}$

Table 6. $p$ values from the non-parametric Kruskal Wallis test comparing the branch length distribution in $L_{2}$ between the studied groups.

$\begin{array}{cccc}L_{2} & \text { Imp L } & \text { Imp Sh } & \text { Prof Rescue } \\ \text { WT } & 8.92 \mathrm{E}-05 & 0.9392 & 0.7884 \\ \text { Imp L } & & 9.04 \mathrm{E}-04 & 0.0014 \\ \text { Imp Sh } & & & 0.9134\end{array}$

Table 7. $p$ values from the non-parametric Kruskal Wallis test comparing the branch length distribution in $L_{4}$ between the studied groups.

$\begin{array}{cccc}L_{4} & \text { Imp L } & \text { Imp Sh } & \text { Prof Rescue } \\ \text { WT } & 3.45 \mathrm{E}-04 & 1.29 \mathrm{E}-04 & 0.1822 \\ \text { Imp L } & & 0.7383 & 0.1238 \\ \text { Imp Sh } & & & 0.1387\end{array}$

\section{$4 \quad$ Likelihood analysis}

For a neuron $n$, we calculated the value of each feature and then compute the likelihood for each group of neurons $\mathrm{X},(\mathrm{X} \in\{\mathrm{WT}, \mathrm{Imp}$, Prof Rescue $\})$. The neuron $n$ is then classified in the group that maximizes the global likelihood. All the classifications present 
in this work were done using the leave one out technique, which consists in classifying an element of the sample that has been removed from the database to perform the learning stage (i.e. the estimation of the models parameters). This maximum likelihood classification provides some assessment about the discriminative properties of the proposed models but is also used to analyze the mixture of feature values between the populations.

Considering our four features to be independent from each other, the global likelihood is given as follows

$$
\begin{gathered}
L\left(\left\{l_{n}, \#_{1, n}-\#_{150, n}, k_{n}, \vec{b}_{n}\right\} \mid n \in \mathrm{X}\right)= \\
L_{l}\left(l_{n} \mid n \in \mathrm{X}\right) L_{s h}\left(\#_{1, n}-\#_{150, n} \mid n \in \mathrm{X}\right) L_{b p}\left(k_{n} \mid n \in \mathrm{X}\right) L_{b l}\left(\vec{b}_{n} \mid n \in \mathrm{X}\right),
\end{gathered}
$$

and the maximum likelihood estimation results

$$
\begin{gathered}
n \in \mathrm{X}_{o} \leftrightarrow \mathrm{X}_{o}=\underset{\mathrm{X}}{\operatorname{argmax}} L\left(\left\{l_{n}, \#_{1, n}-\#_{150, n}, k_{n}, \vec{b}_{n}\right\} \mid n \in \mathrm{X}\right), \\
\mathrm{X}=\{\mathrm{WT}, \text { Imp L, Imp Sh, Prof Rescue }\} .
\end{gathered}
$$

Equation (13) allows to classify each neuron by resemblance to each group considering the four morphological features (main axon length and shape, branch length distribution

\begin{tabular}{|c|c|c|c|c|}
\hline & & \multicolumn{3}{|c|}{ Predicted $(\%)$} \\
\hline & & WT & Imp L & Imp Sh \\
\hline \multirow{4}{*}{ 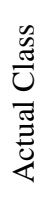 } & WT & 82.6 & 17.4 & 0 \\
\hline & Imp L & 54.5 & 45.5 & 0 \\
\hline & Imp Sh & 19.2 & 3.9 & 76.9 \\
\hline & Prof Rescue & 40 & 26.7 & 33.3 \\
\hline
\end{tabular}
and branch point distribution) and their mathematical models. Table 8 presents the results of the global resemblance analysis.

Table 8. Global likelihood analysis considering the four features.

These results suggest a relevant global difference between neurons belonging to Imp $\mathrm{L}$ and Imp Sh, as well as between WT and Imp Sh; while between WT and Imp L this difference is weaker. More than half of Imp L neurons are likely to be WT while for Imp Sh this proportion is less than $20 \%$. Some WT axons are classified as Imp L but none as Imp Sh. Interestingly, the percentage of Prof Rescue neurons likely to be WT lies in between those percentages for Imp Sh and Imp L. This result points in the direction of a partial rescue of the imp neuron morphology.

To understand how each morphological feature contributes to the results in Table 8 , we carried out the maximum likelihood analysis regarding each of them separately. For the main axon length, as expected from Figure 6, WT neurons are shared between WT and Imp L categories; and Imp L is correspondingly mixed with WT. Imp Sh is completely separated from the rest of the groups (Table 9). Regarding Prof Rescue, our 
results agree with those in Medioni et al. [4] about the main axon length being partially rescued by profilin overexpression. Our study pointed out that $54 \%$ of imp mutant neurons present a conserved main axon length while $46 \%$ are significantly shorter than WT (Section 3.1). Here we show that Prof Rescue neurons are distributed by a 67\% (Imp L +WT) vs. 33\% (Imp Sh), moving the tendency towards a WT phenotype.

Table 9. Likelihood analysis according to the main axon length feature.

\begin{tabular}{|c|c|c|c|c|}
\hline & \multirow{2}{*}{$\mathrm{L}$} & \multicolumn{3}{|c|}{ Predicted $(\%)$} \\
\hline & & WT & Imp L & Imp Sh \\
\hline \multirow{4}{*}{ 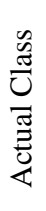 } & WT & 39.1 & 54.4 & 6.5 \\
\hline & $\operatorname{Imp~L}$ & 22.7 & 77.3 & 0 \\
\hline & Imp Sh & 0 & 0 & 100 \\
\hline & Prof Rescue & 6.7 & 60 & 33.3 \\
\hline
\end{tabular}

According to the main axon shape in Table 10, WT and Imp L look again similar and, interestingly, Imp Sh looks more similar to WT than to Imp L. Prof Rescue behavior is opposite to that of Imp Sh.

Table 10. Likelihood analysis according to the main axon shape feature.

\begin{tabular}{|c|c|c|c|c|}
\hline & \multirow{2}{*}{ SH } & \multicolumn{3}{|c|}{ Predicted $(\%)$} \\
\hline & & WT & $\operatorname{Imp} \mathrm{L}$ & Imp Sh \\
\hline \multirow{4}{*}{$\begin{array}{l}\frac{\tilde{y}}{0} \\
\frac{0}{U} \\
\frac{\pi}{\tilde{Z}} \\
\frac{0}{2}\end{array}$} & WT & 54.3 & 43.5 & 2.2 \\
\hline & $\operatorname{Imp~L}$ & 50 & 50 & 0 \\
\hline & Imp Sh & 61.5 & 38.5 & 0 \\
\hline & Prof Rescue & 40 & 60 & 0 \\
\hline
\end{tabular}

Table 11 presents the likelihood analysis results regarding the branch point density. It can be noticed that every group is mainly classified as Imp Sh, which our previous analysis revealed as the most branched group. The reason for this behavior relies on the nature of the model. Even though the means of the distances between branches are different between the biological groups, axons frequently display one or more pairs of branches which are close. Because for close branches the likelihood is maximum for Imp Sh, with a significant difference from the other groups, the presence of near branches automatically classifies a neuron as Imp Sh. Nevertheless, the branch density coherence is respected for each group as the resemblance with Imp Sh is maximum for the most branched group (itself) and is followed in the correct order: WT first, followed by Imp L and Prof Rescue.

Table 11. Likelihood analysis according to the branching point feature.

BP $\quad$ Predicted $(\%)$ 


\begin{tabular}{|c|c|c|c|c|}
\hline & & WT & Imp L & Imp Sh \\
\hline \multirow{4}{*}{ 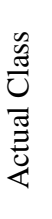 } & WT & 0 & 13 & 87 \\
\hline & Imp L & 13.6 & 18.2 & 68.2 \\
\hline & Imp Sh & 7.7 & 11.5 & 80.8 \\
\hline & Prof Rescue & 6.7 & 26.7 & 66.7 \\
\hline
\end{tabular}

Finally, according to the branch length distribution (Table 12) WT, Imp L and Imp Sh show a higher resemblance to their own groups, suggesting a significant difference between them regarding this feature. Prof Rescue has a slight preference for Imp Sh is understandable as both have the same proportion of branches in $L_{2}$ which is the most abundant group of branches. Nevertheless, its resemblance to WT regarding this feature is notoriously higher than those for $\operatorname{Imp} \mathrm{L}$ and $\operatorname{Imp} \mathrm{Sh}$. This results reveal that profilin overexpression partly rescues branch length distribution -i.e. it presents a bigger proportion of long branches- in addition to the main axon length. Thus Profilin may be involved in the branching process as well.

Table 12. Likelihood analysis according to the branch length distribution feature.

\begin{tabular}{|c|c|c|c|c|}
\hline & \multirow{2}{*}{$3 \mathrm{~L}$} & \multicolumn{3}{|c|}{ Predicted $(\%)$} \\
\hline & & WT & $\operatorname{Imp} \mathrm{L}$ & Imp Sh \\
\hline \multirow{4}{*}{ 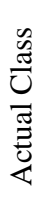 } & WT & 60.9 & 23.9 & 15.2 \\
\hline & $\operatorname{Imp~L}$ & 18.2 & 72.7 & 9.1 \\
\hline & Imp Sh & 15.4 & 30.8 & 53.8 \\
\hline & Prof Rescue & 33.3 & 20 & 46.7 \\
\hline
\end{tabular}

In order to better understand the morphological changes induced by Profilin overexpression in imp mutant axons, we performed the global maximum likelihood analysis

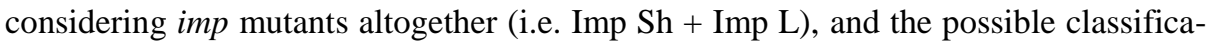
tion groups either altogether (Table 13) or split between Imp L and Imp Sh (Table 14).

Table 13. Global likelihood analysis considering the four features. Imp englobes Imp Sh and Imp L.

\begin{tabular}{|c|c|c|c|}
\hline & & \multicolumn{2}{|c|}{ Predicted $(\%)$} \\
\hline & & WT & Imp \\
\hline \multirow{3}{*}{ 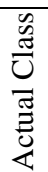 } & WT & 80.4 & 19.6 \\
\hline & Imp & 37.5 & 62.5 \\
\hline & Prof Rescue & 60 & 40 \\
\hline
\end{tabular}

Table 14. Global likelihood analysis considering the four features. Imp is splitted between L and Sh for possible classification groups. 


\begin{tabular}{|c|c|c|c|c|}
\hline & & WT & Imp L & Imp Sh \\
\hline \multirow{3}{*}{ 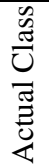 } & WT & 82.6 & 17.4 & 0 \\
\hline & Imp & 35.5 & 23 & 41.5 \\
\hline & Prof Rescue & 40 & 26.7 & 33.3 \\
\hline
\end{tabular}

From the analysis in Table 13, we can highlight that while only $37.5 \%$ of imp mutants present a WT phenotype, $60 \%$ of Profilin rescue neurons exhibit this behavior. Moreover, it is interesting to analyze how Prof Rescue is classified regarding Imp L and Imp Sh (Table 14). The percentage of neurons classified as Imp Sh decreases compared to imp mutants from 42 to $33 \%$ while the tendency for Imp L and WT is increased in Prof Rescue.

Finally a brief comparison can be done regarding our classification results with those in Mottini et al. [21], who also analysed wild type as well as imp mutant gamma neurons. The authors report an 80.4 and $91.7 \%$ of accurate classifications for WT and imp mutants respectively with the ESA curve distance method and 85 and $79.2 \%$ with RTED. It is relevant to highlight that the goal in their work was to discriminate between populations, thus they considered exclusively highly discriminative parameters. On the contrary, our results -80.4 and $62.5 \%$ for WT and Imp respectively- show and value not only the differences but also the existing similarities between phenotypes, considering all the relevant morphological features (including those that may be known as not discriminative). Our work also allows to correlate the conclusions with biological parameters. In addition, our sample size doubles the one used in the cited work.

\section{Discussion}

\subsection{Axon growing rate and branch formation}

The value of $A=1$ indicates that the axon tip diminishes its growing speed only two times before stopping to create a branch, instead of doing it gradually. The first time may be related to the moment when it senses the external guiding cues. Then it continues growing more slowly, which may facilitate other cues detection, until it finally stops, consequence of the second and last speed lost. When this happens, branching material could be accumulated and after some time an interstitial branch is created. An increased value of $p$ may indicate a higher sensibility to external cues as well as an increased concentration of internal cues triggering branching. Another interpretation can be that axons with a defective growing rate (i.e. slower speed, or high $p$ ) are more susceptible to stop independently from external cues, and therefore to branch more.

All the groups present the same value of $A$ indicating that this two-step behavior may be conserved and therefore independent from Imp. Regarding $p$, Imp Sh is significantly more branched than the rest of the groups, including Imp L, even though they have the same genotype. We suggest a correlation between the size of the main axon and the branch density for imp mutants. More interestingly, Prof Rescue axons present the same value of $p$ than Imp L. This suggests that the phenotype presenting high branch density may be rescued by profilin overexpression. 


\subsection{Wild type neurons are mostly differentiated by their branch length distribution}

The global maximum likelihood analysis results in more than $80 \%$ of WT axons to be correctly classified (Table 8, Table 13 and Table 14). Nevertheless, when looking at each particular feature it becomes evident that WT shares most of those with Imp L. Regarding the main axon length (Table 9), 54\% of WT neurons are likely to be Imp L and $43 \%$ for the main axon shape (Table 10). The analysis following the branching point density results in $13 \%$ of WT neurons likely to be Imp L, while no WT neuron was correctly classified. This results are validated by the $p$ values for main axon length and branch length distribution that do not show significant differences between WT and Imp L. We encounter a similar situation regarding the shape model, as between Imp L and WT the amount of significantly different parameters is the minimum of all the group pairs and it is only 12 in 150 .

The maximum likelihood analysis taking only the branch length distribution into account is the sole to correctly classify WT axons (Table 12). While WT and Imp L present both $80 \%$ of branches in $L_{2}$ and $L_{4}$ altogether (Table 5), the difference between them is that WT shows statistically more branches in $L_{4}$ while $\operatorname{Imp} \mathrm{L}$ in $L_{2}$. We can relate our results to those of Tessier and Broadie [20] and Medioni et al. [4]. The first publication reports that a loss of $L_{2}$ branches by a late pruning process occurs in wild type neurons and not in dFMRP mutants (dFMRP is also a profilin regulator) and the second one concludes a defective development of long branches $\left(L_{4}\right)$ in imp mutants.

The maximal percentage of correct classification for WT considering the features separately is $60 \%$ for the branch length distribution (Table 12), followed by 54, 39 and even $0 \%$ corresponding to main axon shape, length and branching point distribution (Table 10, Table 9 and Table 11). Interestingly, the global classification mixing the four features improves these percentages to $80 \%$ (Table 8, Table 13 and Table 14). This suggests that WT neurons are well defined and different from imp mutants but it is necessary to consider all the morphological features together for a correct classification. This also highlights the advantages of our method as it goes beyond a simple statistical analysis, allowing to mix different features as well as to consider each neuron independently.

\subsection{Imp knockdown presents two different phenotypes}

Medioni et al. (2014) [4] reported that imp mutants could either present a conserved main axon length or an aberrant one, with a $~ 50 \%$ of occurrence each. Following these results we applied the k-means automatic algorithm to separate the Imp population in Imp L and Imp Sh, and obtained a 46 vs. $54 \%$ of incidence correspondingly. This bimodal behavior can also be seen in the length distribution (Figure 6). Surprisingly, we have found other relevant morphological differences between this two groups that have not been yet reported in the bibliography. The main one is the branching point distribution, as Imp Sh is significantly more densely branched than Imp L (Table 3 and Table 4). Also, the percentage of branches ranging from 1 to $5 \mu \mathrm{m}$, while aberrant in Imp L, is conserved in Imp Sh (which shows no differences from WT (Table 6)). 
Regarding the global likelihood analysis (Table 8), while less than $20 \%$ of Imp Sh neurons can be considered to have a WT phenotype, 55\% of Imp L do, allowing to conclude that Imp L presents a generally more conserved phenotype. Globally, we conclude that the penetrance of the imp phenotype is $\sim 63 \%$, following our global likelihood analysis (Table 13 and Table 14).

These results are consistent with an essential role of Imp in main axon elongation and branch formation as well as branch elongation during remodelling. Nevertheless, the phenotypical variability within imp mutants (i.e. from globally aberrant to completely WT-like neurons) indicates the existence of other -maybe Imp independentimportant actors with the capability of controlling these processes and neutralize Imp absence; or that the knockdown of the gene is not $100 \%$ efficient.

\subsection{Profilin overexpression partially rescues the main axon length as well as the branch length distribution}

The global likelihood analysis (Table 13) considering Imp altogether shows that Profilin decreases the percentage of imp mutant phenotype from 63 to $40 \%$.

Regarding the main axon length, while aberrant neurons represent $54 \%$ of the Imp population, they represent only 33\% in Prof Rescue (in Prof Rescue 67\% of neurons present a conserved length (WT $+\operatorname{Imp~L)}$ ) and only $33 \%$ do not). Following the branch length distribution resemblance analysis, 33\% of Prof Rescue neurons are classified as WT and represent the second maximum percentage after WT itself (only 18 and 15\% correspond to Imp L and Sh, respectively). Looking at the $p$ values between branch length categories (Table 6 and Table 7), we can conclude that Profilin rescues the late pruning of small branches [20] showing a conserved percentage of $L_{2}$ branches and also allows to develop more long branches. Even though the percentage of branches in $L_{4}$ is slightly smaller for Prof Rescue than WT (Table 5), this difference is not significant in the statistical tests, suggesting a conserved percentage of long branches in Prof Rescue which is not seen in Imp Sh nor in Imp L.

Finally, regarding the global likelihood analysis considering Imp L and Imp Sh separately (Table 14), we conclude that Profilin rescue diminishes the general morphological aberration, as it moves the tendency towards WT and Imp L phenotypes and lowers the percentage of neurons with an Imp Sh phenotype.

This study suggests that Profilin is also involved in branch formation and elongation, in addition to main axon elongation during remodelling. Nevertheless, because the phenotypical rescue is not complete, we can conclude either that its regulation by Imp is still an essential step in remodelling or that other Imp targets are also essential in these processes; or -most probably- both simultaneously.

\section{Conclusions}

In this work we proposed a framework to compare neuron groups based on their morphology. Our procedure consists in applying probabilistic models to describe the behavior of selected morphological features (i.e. main axon length and shape as well as branch length and density), the estimation from data of the associated parameters and a 
resemblance analysis combined with statistical tests. We applied this framework to understand the effects of imp knockdown -as well as its rescue by Profilin- in Drosophila gamma adult neuron morphology. The similarities and differences we are able to highlight between wild type and mutant neurons allow to better understand the role of Imp and Profilin during axonal remodelling, particularly on axon elongation and branch formation.

We propose that this method consisting in feature selection, model application and likelihood analysis could be applied to any case of study between species where similarities are as important as differences. We can also conclude that the study of individuals is relevant and more enriching than just population analysis driven by ordinary statistics. Finally, we highlight the importance of combining different features to achieve a global result.

\section{Acknowledgements}

This work was supported by the French Government (National Research Agency, ANR) through the «Investments for the Future » LABEX SIGNALIFE: program reference \# ANR-11-LABX-0028-01.

All the authors are within Morpheme (a joint team between Inria CRI-SAM, I3S and IBV).

\section{Appendix}

From Equation 6 we can express $\mu_{\mathrm{k}}(A+1, p)$ as

$$
\mu_{\mathrm{k}}(A+1, p)=\sum_{k} k\left(\begin{array}{c}
k-1 \\
A+1
\end{array}\right) p^{A+2}(1-p)^{k-A-2} .
$$

Using

$$
\left(\begin{array}{c}
k-1 \\
A
\end{array}\right)+\left(\begin{array}{c}
k-1 \\
A+1
\end{array}\right)=\left(\begin{array}{c}
k \\
A+1
\end{array}\right)
$$

Equation 14 can be rewritten as

$$
\begin{gathered}
\mu_{\mathrm{k}}(A+1, p)=\sum_{k} k\left(\begin{array}{c}
k \\
A+1
\end{array}\right) p^{A+2}(1-p)^{k-A-2} \\
-\sum_{k} k\left(\begin{array}{c}
k-1 \\
A
\end{array}\right) p^{A+2}(1-p)^{k-A-2} .
\end{gathered}
$$

Taking out $\frac{p}{1-p}$ as a common factor from the second sum in Equation (16), we obtain

$$
\begin{gathered}
\sum_{k} k\left(\begin{array}{c}
k-1 \\
A
\end{array}\right) p^{A+2}(1-p)^{k-A-2}= \\
\frac{p}{1-p} \sum_{k} k\left(\begin{array}{c}
k-1 \\
A
\end{array}\right) p^{A+1}(1-p)^{k-A-1}=\frac{p}{1-p} \mu_{\mathrm{k}}(A, p) .
\end{gathered}
$$

Similarly, the first sum in (16) can be worked out to obtain 


$$
\mu_{\mathrm{k}}(A+1, p)=\frac{1}{1-p} \mu_{\mathrm{k}}(A+1, p)-\frac{1}{1-p} .
$$

From (17) and (18) we finally obtain

$$
\mu_{\mathrm{k}}(A, p)=\frac{A}{p}
$$

From Equations (7) and (19) we can express

$$
\sigma_{\mathrm{k}}^{2}(A, p)=\sum_{k} k^{2}\left(\begin{array}{c}
k-1 \\
A
\end{array}\right) p^{A+1}(1-p)^{k-A-1}-\frac{1}{p^{2}} A^{2},
$$

from where

$$
\sigma_{\mathrm{k}}^{2}(A+1, p)=\sum_{k} k^{2}\left(\begin{array}{c}
k-1 \\
A+1
\end{array}\right) p^{A+2}(1-p)^{k-A-2}-\frac{1}{p^{2}}(A+1)^{2} .
$$

Equation (21) can be rewritten using Equation (15) to get $\sigma^{2}{ }_{\mathrm{k}}(A+1, p)$ :

$$
\begin{gathered}
\sigma_{\mathrm{k}}^{2}(A+1, p)=\sum_{k} k^{2}\left(\begin{array}{c}
k \\
A+1
\end{array}\right) p^{A+2}(1-p)^{k-A-2} \\
-\sum_{k} k^{2}\left(\begin{array}{c}
k-1 \\
A
\end{array}\right) p^{A+2}(1-p)^{k-A-2}-\frac{1}{p^{2}}(A+1)^{2},
\end{gathered}
$$

Working out Equation (22) similarly to Equation (16) we finally get

$$
\sigma_{\mathrm{k}}^{2}(A, p)=\frac{(1-p) A}{p^{2}}
$$

\section{References}

1. Xie, Z., Huang, C., Ci, B., Wang, L., and Zhong, Y., 2013. Requirement of the combination of mushroom body $\gamma$ lobe and $\alpha / \beta$ lobes for the retrieval of both aversive and appetitive early memories in Drosophila. Learning \& Memory,20(9), 474-481.

2. Redt-Clouet, C et al., 2012. Mushroom body neuronal remodelling is necessary for shortterm but not for long-term courtship memory in Drosophila. European Journal of Neuroscience, 35(11), 1684-1691.

3. Williams, D. W., and Truman, J. W., 2005. Remodeling dendrites during insect metamorphosis. Journal of neurobiology, 64(1), 24-33.

4. Medioni, C., Ramialison, M., Ephrussi, A., and Besse, F., 2014. Imp promotes axonal remodeling by regulating profilin mRNA during brain development. Current Biology, 24(7), 793-800.

5. Luo, L., 2002. Actin cytoskeleton regulation in neuronal morphogenesis and structural plasticity. Annual review of cell and developmental biology, 18(1), 601-635.

6. Schlüter, K., Jockusch, B. M., and Rothkegel, M., 1997. Profilins as regulators of actin dynamics. Biochimica et Biophysica Acta (BBA)-Molecular Cell Research, 1359(2), 97-109.

7. Verheyen, E. M., and Cooley, L., 1994. Profilin mutations disrupt multiple actin-dependent processes during Drosophila development. Development, 120(4), 717-728.

8. Kong, J. H., Fish, D. R., Rockhill, R. L., and Masland, R. H., 2005. Diversity of ganglion cells in the mouse retina: unsupervised morphological classification and its limits. J. of Comp. Neurology, 489(3), 293-310. 
9. Guerra, L., McGarry, L. M., Robles, V., Bielza, C., Larranaga, P., \& Yuste, R., 2011. Comparison between supervised and unsupervised classifications of neuronal cell types: a case study. Developmental neurobiology, 71(1), 71-82.

10. López-Cruz, P. L., Larrañaga, P., DeFelipe, J., and Bielza, C., 2014. Bayesian network modeling of the consensus between experts: An application to neuron classification. Int. J. of Approx. Reasoning, 55(1), 3-22.

11. Mottini A, Descombes X., and Besse F., 2014. From Curves to Trees: A Tree-like Shapes Distance Using the Elastic Shape Analysis Framework. Neuroinformatics, 13, 175-191.

12. Wu, J. S., and Luo, L., 2006. A protocol for mosaic analysis with a repressible cell marker (MARCM) in Drosophila. Nature protocols, 1(6), 2583-2589.

13. Schindelin, J et al, 2012. Fiji: an open-source platform for biological-image analysis. Nature meth.9(7), 676-682.

14. Myatt, D. R., Hadlington, T., Ascoli, G. A., and Nasuto, S. J., 2012. Neuromantic-from semi-manual to semi-automatic reconstruction of neuron morphology. Frontiers in neuroinformatics, 6 .

15. Mottini, A., Descombes, X., Besse, F., and Pechersky, E., 2014. Discrete stochastic model for the generation of axonal trees. In EMBS, 6814-6817.

16. Kemeny, J. G., and Snell, J. L., 1960. Finite markov chains, vol. 356. van Nostrand, $1^{\text {st }}$ edition.

17. Keller, M.T. and Trotter, W.T., 2015. Applied Combinatorics. Georgia

18. Szebenyi, G., Callaway, J. L., Dent, E. W., and Kalil, K., 1998. Interstitial branches develop from active regions of the axon demarcated by the primary growth cone during pausing behaviours. The Journal of neuroscience, 18(19), 7930-7940.

19. Forbes, C., Evans, M., Hastings, N., and Peacock, B., 2011. Statistical distributions, John Wiley \& Sons, $4^{\text {th }}$ edition.

20. Tessier, C. R., and Broadie, K., 2008. Drosophila fragile X mental retardation protein developmentally regulates activity-dependent axon pruning. Development, 135(8), 1547-1557.

21. Mottini, A., Descombes, X., and Besse, F., 2013. Tree-like shapes distance using the elastic shape analysis framework. In British Machine Vision Conference. 